\title{
Spectacular Effect of Massive Facial Angiofibromas Removal With a Carbon Dioxide Laser as a Manifestation of a Tuberous Sclerosis Complex
}

\author{
Adam Borzęcki $^{\circledR}$, Katarzyna Chyl-Surdacka $^{\oplus}$, Monika Turska $^{*(®)}$ \\ 'Department of Dermatology, Non-Public Health Care Center Med-Laser, Młyńska 14A Str. 20-406 Lublin
}

\author{
*Correspondence to \\ Monika Turska, \\ Department of Dermatology, \\ Non-Public Health Care Center \\ Med-Laser, Młyńska 14A Str. \\ 20-406 Lublin \\ Tel: 0048815329090 \\ Email: turskamk@gmail.com \\ Received: 8 December 2020 \\ Accepted: 16 February 2021 \\ Published online 15 June 2021

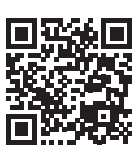

\section{Introduction}

Tuberous sclerosis complex (TSC), also called BournevillePringle disease, is a rare multisystem genetic disease with an incidence estimated at $1 / 6000$ to $1 / 10000$ live births and $1 / 20000$ in the whole population..$^{1-4}$ Despite the fact that the rate of spontaneous mutations is high (70\%), it is described to have an autosomal dominant pattern of inheritance. The most common are mutations in the genes encoding either hamartin (TSC1) or tuberin (TSC2). ${ }^{1,5}$

In healthy people, hamartin and tuberin proteins are involved in regulating cell division by inhibiting the Pi3K-Akt-mTOR pathway. In TSC, mutations in the TSC1 and TSC2 genes with a loss of their function induce an increase in the activity of the described pathway, the formation of uncontrolled cell division and cancer tumors. ${ }^{1,4,6}$ The mutation of the TSC2 gene is about four times more common in cases of spontaneous mutations. ${ }^{1}$ The course of the disease, the onset of symptoms and the number of organs involved vary between patients. ${ }^{2,6,7}$ The mutations of hamartin and tuberin consequently lead to neurological, cardiological, nephrological, ophthalmic, pulmonary and dermatological disorders. Although skin lesions present in patients with TSC are not the most serious health problems, they may turn out to be a significant aesthetic defect which significantly affects patients' quality of life.

\section{Case Presentation}

A 64-year-old man with TSC diagnosed in childhood was admitted to the Department of Dermatology Non-Public Health Care Center 'Med-Laser' in Lublin due to the presence of numerous, massive nodules of the chin and paranasal folds (Figure 1A).

The patient reported the formation of the first skin lesions in his childhood, while he noticed accelerated growth during the last three years when the ischemic stroke occurred.

According to the patient's medical history, he suffered from renal angiomyolipomas, multiple renal cysts and chronic kidney disease. In addition, he was treated for hypertension, heart failure, and normocytic anaemia. About three years before the admission, he started taking anticoagulants due to a previous pulmonary embolism and an ischemic stroke.

Moreover, during a physical examination, fibrous plaques were found on the forehead and the parietal area of the scalp, accompanied by numerous ungual fibromas of both feet (Figure 1B, 1C).

A skin biopsy was taken from nodules located on his chin. Histopathological examination confirmed angiofibromas. Surgical treatment was not proposed due to the multiple chronic conditions. A significant aesthetic defect, food intake difficulty, development of nodular lesions, inflammation and local bleeding made laser treatment the most suitable option. 


\section{Laser Procedure}

Information about the techniques of analgesia, the procedure and photographic documentation was administered once the written consent was obtained.

Local anesthesia with articaine and adrenaline solution was used. The carbon dioxide $\left(\mathrm{CO}_{2}\right)$ surgical laser machine (Gmerk) was applied. Firstly, the lesions were cut in continuous mode with a pulse providing 20 watts of power, with pulse lengths of 500 microseconds. Afterwards, evaporation was applied in ultrapulse mode to generate pulses of $10 \mathrm{~W}$ of power, each pulse length of 350 microseconds. During all laser procedures the eyes of both patient and medical staff were protected by laser safety glasses. In addition, the Jumbo filter trolley was used for absorbing and filtering gases and vapours.

Single lesions were gradually removed by undercutting peduncles and coagulation of bleeding foci (Figure 2A, $2 \mathrm{~B})$. After the first stage, in which the largest number of nodules was removed, prophylactic antibiotic therapy with oral metronidazole was used. In total, 4 laser procedures were performed. The intervals between laser treatments were 3 to 4 weeks. TThe effect of applied therapy, which is presented in Figure 2D, was spectacular. A 6-month follow-up examination revealed that each and every angiofibroma on the chin and nasolabial folds was removed, and no scarring or hypopigmentation occurred. In addition, no regrowth was noticed. The patient remains under constant dermatological, nephrological, neurological and cardiological control.

\section{Discussion}

Cutaneous manifestations of TSC are crucial not only for medical doctors but also for patients. They are an important, stigmatizing aesthetic defect which often impede everyday functioning and social interactions. As a result, patient's physical and psychological status and the quality of life decline. Facial angiofibromas seem to be the most troublesome. According to scientific data, surgical resection and laser ablation are the primarily used techniques for the treatment of such lesions. ${ }^{1,4}$ Different removal techniques were considered, relying on the size and location of angiofibromas. Papadavid et al have demonstrated excellent effects in flattening the fibrous and protuberant angiofibromas with the use of the $\mathrm{CO}_{2}$ laser in comparison to the flashlamp-pumped pulsed dye laser. ${ }^{8}$ However, hypertrophic scarring was noticed as a short-term side effect of the procedure. In those cases, the usage of intralesional corticosteroids has shown considerable improvement. In our case, no scarring was observed. However, long-term observation of our patient

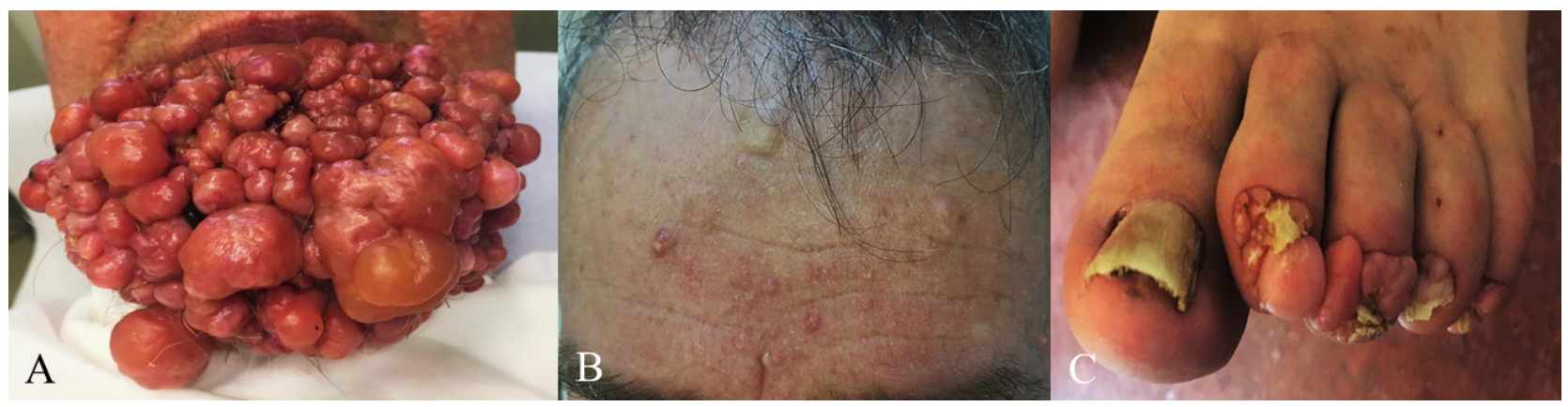

Figure 1. Clinical Features Before Treatment. (A) Angiofibromas before treatment, (B) Fibrous cephalic plaques, (C) Ungual fibromas.

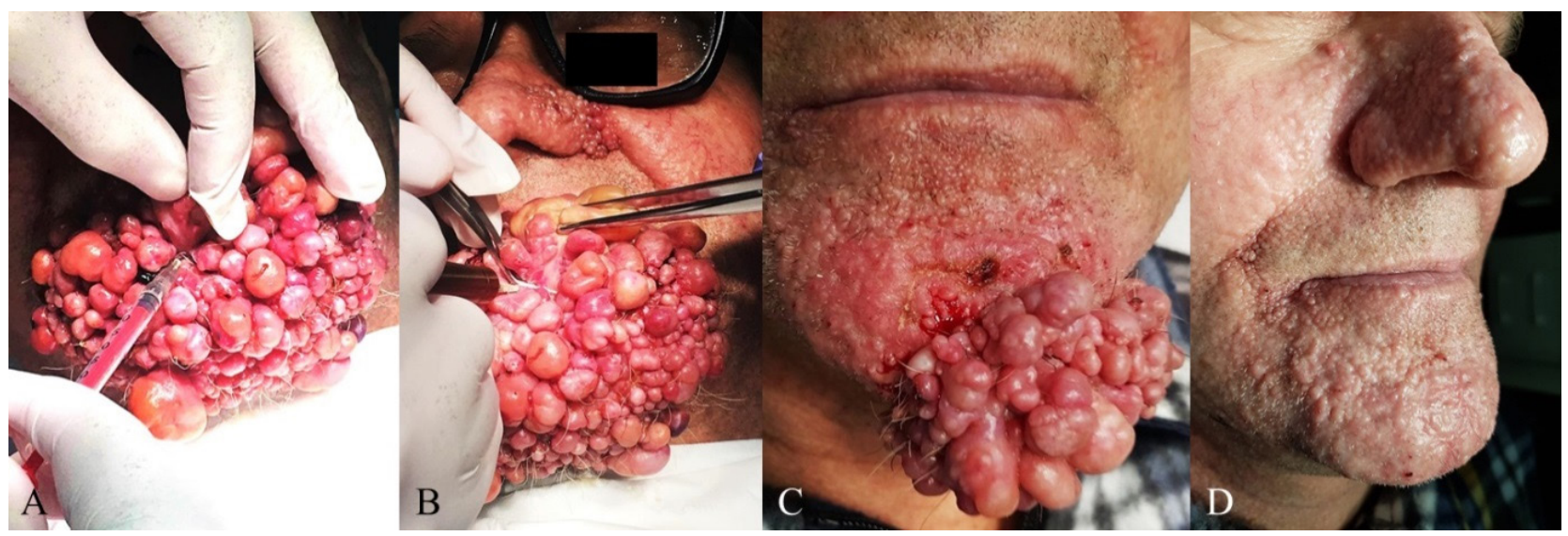

Figure 2. Laser Treatment Procedure. (A) Local anesthesia, (B) Removal of single angiofibromas with a $\mathrm{CO}_{2}$ laser, (C) First effects of laser therapy (D) Final effects of laser therapy. 
is required. On the other hand, Faisal et al, based on their own observations between the years 2009 and 2015 and the literature review, have reported almost no side effects after $\mathrm{CO}_{2}$ laser treatment and recommended ablative laser treatment as a well-tolerated and effective procedure. ${ }^{9}$ In less severe cases of angiofibromas, the fractional $\mathrm{CO}_{2}$ laser alone or combined with pinpoint electrosurgery, pulseddye laser treatment, and ablative fractional resurfacing can be used. Weiss et al presented such a combination in three cases with good results and reduction of scarring. ${ }^{10}$ Ma et al described the effectiveness of Nd:YAG laser at a wavelength of $1064 \mathrm{~nm}$ with five to eight treatment sessions. Immediate improvement was noticed in all twelve cases. However, a slight recurrence of angiofibromas was noted after twelve months. ${ }^{11}$

Similar to the $\mathrm{CO}_{2}$ laser, the erbium:yttrium-aluminumgarnet (Er:YAG) laser, with a wavelength of $2940 \mathrm{~nm}$, targets water in the skin tissue leading to the vaporization of superficial cells. Although short impulses of waves are absorbed more efficiently by water in tissues than the $\mathrm{CO}_{2}$ laser, less prevention of bleeding is achieved. In addition, the Er:YAG laser has different penetration depths in comparison to the $\mathrm{CO}_{2}$ laser; hence it is used mostly in cases that require superficial ablation only.

Although different laser types may be helpful in such conditions, the $\mathrm{CO}_{2}$ laser seems to be the most effective due to its ablative indications and safety. A huge advantage of the $\mathrm{CO} 2$ laser is its ability to work in two modes: continuous and pulsed. It allows medical doctors to first cut the tissue layer by layer and then cut chosen structures like a knife. Because of high temperatures, it is able to coagulate vessels, which was extremely important in our case. As a result, haemorrhage was significantly reduced. Last but not least, it is very precise. Therefore, no damage to surrounding tissues occurred and the healing process was facilitated.

It should be mentioned that the management of angiofibromas seems challenging, especially in the presence of a large number and size of lesions, in pediatric populations and among people with severe intellectual disabilities, who often require general anesthesia.

To sum up, laser treatment of facial skin angiofibromas in patients with TSC appears to be a beneficial therapeutic option which is worth considering, especially when surgical treatment is contraindicated or before the invention of alternative therapeutic possibilities.

\section{Conflict of Interests}

All authors certify that they have no affiliations with or involvement in any organization or entity with any financial interest or non-financial interest in the subject matter discussed in this manuscript.

\section{Ethical consideration:}

Written informed consent was obtained from the patient for publication of this report.

\section{Acknowledgments}

No source of financial grants and other funding was provided.

\section{References}

1. Randle SC. Tuberous Sclerosis Complex: A Review. Pediatr Ann. 2017;46(4):e166-e71. doi: 10.3928/1938235920170320-01

2. Annear NMP, Appleton RE, Bassi Z, Bhatt R, Bolton PF, Crawford P, et al. Tuberous Sclerosis Complex (TSC): Expert Recommendations for Provision of Coordinated Care. Front Neurol. 2019;10(1116). doi: 10.3389/ fneur.2019.01116

3. Bakić M, Ratković M, Gledović B, Vujović B, Radunović D, Babić V, et al. Cutaneous Manifestations of Tuberous Sclerosis. Acta Dermatovenerol Croat. 2018; 26(1):73-4.

4. Wataya-Kaneda M, Uemura M, Fujita K, Hirata H, Osuga K, Kagitani-Shimono K, et al. Tuberous sclerosis complex: Recent advances in manifestations and therapy. Int J Urol. 2017;24(9):681-91. doi: 10.1111/iju.13390

5. Tsang SH, Sharma T. Tuberous Sclerosis. Adv Exp Med Biol. 2018; 1085:205-7. doi: 10.1007/978-3-319-95046-4_43

6. Curatolo P, Maria BL. Tuberous sclerosis. Handb Clin Neurol. 2013; 111:323-31. doi: 10.1016/B978-0-444-528919.00038-5

7. MacKeigan JP, Krueger DA. Differentiating the mTOR inhibitors everolimus and sirolimus in the treatment of tuberous sclerosis complex. Neuro Oncol.2015;17(12):15509. doi: 10.1093/neuonc/nov152

8. Papadavid E, Markey A, Bellaney G, Walker NPJ. Carbon dioxide and pulsed dye laser treatment of angiofibromas in 29 patients with tuberous sclerosis. $\mathrm{Br} \mathrm{J} \mathrm{Dermatol.}$ 2002;147(2):337-42. doi: 10.1046/j.1365-2133.2002.04968.x

9. Ali FR, Mallipeddi R, Craythorne EE, Sheth N, AlNiaimi F. Our experience of carbon dioxide laser ablation of angiofibromas: Case series and literature review. J Cosmet Laser Ther. 2016 Nov;18(7):372-375. doi: 10.1080/14764172.2016.1197403

10. Weiss ET, Geronemus RG. New technique using combined pulsed dye laser and fractional resurfacing for treating facial angiofibromas in tuberous sclerosis. Lasers Surg Med. 2010;42(5):357-60. doi: 10.1002/lsm.20939.

11. Ma G, Wu P, Lin X, Chen H, Li W, Hu X, et al. Nd:YAG laser for „fractional“ treatment of angiofibromas. Int J Dermatol. 2014;53(5):638-42. doi: 10.1111/ijd.12384 